\title{
Approximations for the Bessel and Struve Functions
}

\author{
By J. N. Newman
}

\begin{abstract}
Polynomials and rational-fraction approximations with minimax accuracy are presented for the Bessel functions of the first and second kind of orders zero and one, and for the Struve functions of the same order. The accuracy of these approximations is consistent with typical single-precision computations. The results for the Bessel functions improve upon those in Abramowitz and Stegun [1].
\end{abstract}

1. Introduction. In three-dimensional theories of water wave interactions with floating or submerged bodies, the oscillatory wave-like portion of the relevant Green function can be expressed in terms of Bessel and Struve functions with real arguments. Since problems in this field are solved frequently by boundary-integral methods where the kernel of the resulting integral equation must be evaluated $10^{6}$ to $10^{8}$ times for a given body, efficient approximations are required for these special functions.

Single-precision accuracy is generally sufficient in the above context, with a fixed absolute error. Segmented polynomial approximations, derived originally by Allen [2], are given for the Bessel functions $J_{0}, J_{1}, Y_{0}$, and $Y_{1}$ in Section 9.4 of [1]. For the segment $0 \leqslant x \leqslant 3$, polynomials of sixth degree in the variable $(x / 3)^{2}$ are used. These correspond in form to the truncated ascending power series for each function.

For the complementary segment $x \geqslant 3$, Allen's approximations for the modulus and phase of the Hankel function $J_{n}+i Y_{n}$ are polynomials of sixth degree in the inverse variable $(3 / x)$. This form is surprising, since only even powers of the inverse variable occur in the asymptotic expansion for the modulus, and odd powers for the phase. The disparate magnitude of Allen's polynomial coefficients is further evidence that the form assumed is not optimum; for example, the first odd coefficient in the approximation for each modulus is four orders of magnitude smaller than the next even coefficient.

The present work was limited initially to providing suitable approximations for the Struve functions $\mathbf{H}_{0}$ and $\mathbf{H}_{1}$ to be used in conjunction with the Bessel function approximations in [1]. New approximations also have been derived for the Bessel functions, after observing that the polynomials in [1] could be improved for both subdomains of $x$.

High-accuracy Chebyshev expansions are given by Luke [3] for the Bessel and Struve functions. For the Bessel functions, similar results are given by Clenshaw [4] and minimax rational-fraction approximations are tabulated by Hart et al. [5].

Received April 25, 1983; revised March 1, 1984.

1980 Mathematics Subject Classification. Primary 65D20. 
However, the lower segment extends from $x=0$ to 8 in all of these approximations, with the consequence that polynomials of relatively high degree are required for single-precision accuracy. Thus, optimum polynomial approximations must be rederived in more appropriate subintervals for single-precision use.

The present results have been developed using an IBM PC microcomputer with double-precision internal accuracy of $17 \mathrm{~S}$.

2. Polynomial Approximations for $0 \leqslant x \leqslant 3$. It is straightforward to derive Chebyshev expansions from the ascending power series for $J_{n}$ and the regular part of $Y_{n}$. The coefficients in Table 1 follow by truncation of these expansions and conversion to ordinary polynomials. The forms adopted here for $J_{0}, J_{1}$, and $Y_{0}$ are identical to Allen's approximations [1, Section 9.4, Eqs. 1, 2, 4] but differences may be noted in the coefficients, typically in the fifth or sixth decimal place. The form adopted here for $Y_{1}$ is modified to correspond with the ascending series in odd powers of the argument. In all cases the maximum error is significantly less than that reported in [1].

Analogous polynomials with one less term are listed in Table 2. Similar approximations for the Struve functions $\mathbf{H}_{0}(x)$ and $\mathbf{H}_{1}(x)$ are given in Table 3.

\section{TABLE 1}

Polynomial approximations for $0 \leqslant x \leqslant 3$

$$
\begin{aligned}
J_{0}(x)= & 0.999999999 \\
& -2.249999879(x / 3)^{2} \\
& +1.265623060(x / 3)^{4} \\
& -0.316394552(x / 3)^{6} \\
& +0.044460948(x / 3)^{8} \\
& -0.003954479(x / 3)^{10} \\
& +0.000212950(x / 3)^{12} \\
& +\varepsilon(x) \\
& |\varepsilon|<1.9 \mathrm{E}-09
\end{aligned}
$$

$$
\begin{aligned}
J_{1}(x) / x= & 0.500000000 \\
& -0.562499992(x / 3)^{2} \\
& +0.210937377(x / 3)^{4} \\
& -0.039550040(x / 3)^{6} \\
& +0.004447331(x / 3)^{8} \\
& -0.000330547(x / 3)^{10} \\
& +0.000015525(x / 3)^{12} \\
& +\varepsilon(x) \\
& |\varepsilon|<1.2 \mathrm{E}-09
\end{aligned}
$$

$$
\begin{aligned}
Y_{0}(x)= & (2 / \pi) \ln (x / 2) J_{0}(x) \\
& +0.367466907 \\
& +0.605593797(x / 3)^{2} \\
& -0.743505078(x / 3)^{4} \\
& +0.253005481(x / 3)^{6} \\
& -0.042619616(x / 3)^{8} \\
& +0.004285691(x / 3)^{10} \\
& -0.000250716(x / 3)^{12} \\
& +\varepsilon(x) \\
& |\varepsilon|<3.3 \mathrm{E}-09
\end{aligned}
$$

$$
\begin{aligned}
Y_{1}(x)= & (2 / \pi)\left(\ln (x / 2) J_{1}(x)-1 / x\right) \\
& +0.073735531(x / 3) \\
& +0.722769344(x / 3)^{3} \\
& -0.438896337(x / 3)^{5} \\
& +0.104320251(x / 3)^{7} \\
& -0.013637596(x / 3)^{9} \\
& +0.001125970(x / 3)^{11} \\
& -0.000056455(x / 3)^{13} \\
& +\varepsilon(x) \\
& |\varepsilon|<7.1 \mathrm{E}-10
\end{aligned}
$$


TABLE 2

Simplified polynomial approximations for $0 \leqslant x \leqslant 3$

$$
\begin{aligned}
J_{0}(x)= & 0.99999990 \\
& -2.24999239(x / 3)^{2} \\
& +1.26553572(x / 3)^{4} \\
& -0.31602189(x / 3)^{6} \\
& +0.04374224(x / 3)^{8} \\
& -0.00331563(x / 3)^{10} \\
& +\varepsilon(x) \\
& |\varepsilon|<1.1 \mathrm{E}-07
\end{aligned}
$$

$$
\begin{aligned}
J_{1}(x) / x= & 0.49999999 \\
& -0.56249945(x / 3)^{2} \\
& +0.21093101(x / 3)^{4} \\
& -0.03952287(x / 3)^{6} \\
& +0.00439494(x / 3)^{8} \\
& -0.00028397(x / 3)^{10} \\
& +\varepsilon(x) \\
& |\varepsilon|<1.1 \mathrm{E}-08
\end{aligned}
$$

$$
\begin{aligned}
Y_{0}(x)= & (2 / \pi) \ln (x / 2) J_{0}(x) \\
& +0.36746703 \\
& +0.60558498(x / 3)^{2} \\
& -0.74340225(x / 3)^{4} \\
& +0.25256673(x / 3)^{6} \\
& -0.04177345(x / 3)^{8} \\
& +0.00353354(x / 3)^{10} \\
& +\varepsilon(x) \\
& |\varepsilon|<2.9 \mathrm{E}-07
\end{aligned}
$$$$
\begin{aligned}
Y_{1}(x)= & (2 / \pi)\left(\ln (x / 2) J_{1}(x)-1 / x\right) \\
& +0.07373571(x / 3) \\
& +0.72276433(x / 3)^{3} \\
& -0.43885620(x / 3)^{5} \\
& +0.10418264(x / 3)^{7} \\
& -0.01340825(x / 3)^{9} \\
& +0.00094249(x ? 3)^{11} \\
& +\varepsilon(x) \\
& |\varepsilon|<1.6 \mathrm{E}-08
\end{aligned}
$$

TABLE 3

Struve function approximations for $0 \leqslant x \leqslant 3$

$$
\begin{aligned}
\mathbf{H}_{0}(x)= & 1.909859164(x / 3) \\
& -1.909855001(x / 3)^{3} \\
& +0.687514637(x / 3)^{5} \\
& -0.126164557(x / 3)^{7} \\
& +0.013828813(x / 3)^{9} \\
& -0.000876918(x / 3)^{11} \\
& +\varepsilon(x) \\
& |\varepsilon|<1.2 \mathrm{E}-08
\end{aligned}
$$$$
\begin{aligned}
\mathbf{H}_{1}(x)= & 1.909859286(x / 3)^{2} \\
& -1.145914713(x / 3)^{4} \\
& +0.294656958(x / 3)^{6} \\
& -0.042070508(x / 3)^{8} \\
& +0.003785727(x / 3)^{10} \\
& -0.000207183(x / 3)^{12} \\
& +\varepsilon(x) \\
& |\varepsilon|<2.5 \mathrm{E}-09
\end{aligned}
$$

3. Polynomial Approximations for $x \geqslant 3$. Following the notation of [1], the modulus and phase of the Hankel function are defined by

$$
J_{n}(x)+i Y_{n}(x)=x^{-1 / 2} f_{n} \exp \left(i \theta_{n}\right)
$$

Guided by the corresponding asymptotic approximations for large (positive) $x$, the Chebyshev expansions for the modulus and phase can be combined in the form

$$
f_{n}(|x|)+\operatorname{sgn}(x)\left[\theta_{n}(|x|)-|x|+(2 n+1) \pi / 4\right]=\sum_{m=0}^{\infty} C_{n m} T_{m}(3 / x)
$$


in the extended range $-1 \leqslant(3 / x) \leqslant 1$, with $m$ even or odd for the respective separate expansions. Here $T_{n}(z)$ is the Chebyshev polynomial, and the primed summation indicates that the term $m=0$ is multiplied by $1 / 2$.

The numerical procedure used to determine the coefficients $C_{n m}$ is based on Miller's backward recursion for the Bessel functions, and evaluation of the Chebyshev coefficients by Clenshaw's algorithm [6]. The resulting sequence of Chebyshev coefficients is monotonic in magnitude, and can be truncated effectively to yield the ordinary polynomials in Table 4 . Here the errors are essentially the same as those of Allen, but one less term is required as a consequence of using the more efficient even or odd power series for the modulus and phase, respectively. These polynomial approximations result in absolute errors less than $3 E-08$ for each of the four Bessel functions, throughout the range $x \geqslant 3$.

TABLE 4

Polynomial approximations of Bessel functions for $x \geqslant 3$

$$
\begin{aligned}
& f_{0}=0.79788454 \quad \theta_{0}=x-\pi / 4 \\
& -0.00553897(3 / x)^{2} \quad-0.04166592(3 / x) \\
& +0.00099336(3 / x)^{4} \quad+0.00239399(3 / x)^{3} \\
& -0.00044346(3 / x)^{6} \quad-0.00073984(3 / x)^{5} \\
& +0.00020445(3 / x)^{8} \quad+0.00031099(3 / x)^{7} \\
& -0.00004959(3 / x)^{10} \quad-0.00007605(3 / x)^{9} \\
& +\varepsilon(x)+\varepsilon(x) \\
& |\varepsilon|<1.8 \mathrm{E}-08 \quad|\varepsilon|<5.1 \mathrm{E}-08 \\
& f_{1}=0.79788459 \quad \theta_{1}=x-3 \pi / 4 \\
& +0.01662008(3 / x)^{2} \quad+0.12499895(3 / x) \\
& -0.00187002(3 / x)^{4} \quad-0.00605240(3 / x)^{3} \\
& +0.00068519(3 / x)^{6} \quad+0.00135825(3 / x)^{5} \\
& -0.00029440(3 / x)^{8} \quad-0.00049616(3 / x)^{7} \\
& +0.00006952(3 / x)^{10}+0.00011531(3 / x)^{9} \\
& +\varepsilon(x) \\
& |\varepsilon|<2.5 \mathrm{E}-08 \quad|\varepsilon|<7.3 \mathrm{E}-08
\end{aligned}
$$

A similar procedure may be followed for the Struve functions, with evaluation from running sums of $J_{n}$ during the backward recursion and combining of the odd/even Chebyshev expansions for the respective functions $\mathbf{H}_{0}(x)-Y_{0}(x)$ and $\mathbf{H}_{1}(x)-Y_{1}(x)$ based on the respective asymptotic expansions. However 20 terms are required for an accuracy of $4 \mathrm{E}-08$, corresponding to polynomials for each Struve function with 10 or 11 terms. In view of their complexity these results have been deleted in favor of the more efficient rational-fraction approximations to follow.

4. Rational-Fraction Approximations for $x \geqslant 3$. A variant of Maehly's algorithm [7] has been used to derive minimax rational-fraction approximations for $x \geqslant 3$, with the error curves leveled to $3 \mathrm{~S}$. Three terms in each numerator and denominator yield ultimate maximum errors in the Bessel functions less than $2 \mathrm{E}-08$. The coefficients 
are listed in Table 5, where the function identified by the column heading is approximated in accordance with the rational fractions

$$
f_{n}(x)=\left(a_{0}+a_{1} / x^{2}+a_{2} / x^{4}\right) /\left(1+b_{1} / x^{2}+b_{2} / x^{4}\right)+\varepsilon(x),
$$

(4) $\quad \theta_{n}(x)-x+(2 n+1) \pi / 4$

$$
=\left(a_{0}+a_{1} / x^{2}+a_{2} / x^{4}\right) /\left(x+b_{1} / x+b_{2} / x^{3}\right)+\varepsilon(x) .
$$

TABLE 5

Coefficients of the rational fractions (3)-(4)

$\begin{array}{ccccc} & f_{0} & \theta_{0}-x+\pi / 4 & f_{1} & \theta_{1}-x+3 \pi / 4 \\ a_{0} & 0.79788454 & -0.12499967 & 0.79788459 & 0.37499947 \\ a_{1} & 5.46272781 & -1.07437411 & 4.76650390 & 2.77870488 \\ a_{2} & 3.02562477 & -0.75853664 & 2.58896576 & 1.39381402 \\ & & & & \\ b_{1} & 6.90899779 & 9.11511321 & 5.78645312 & 7.84700458 \\ b_{2} & 4.12217805 & 9.19906287 & 2.35033517 & 6.19124657 \\ |\varepsilon|< & 2.1 \mathrm{E}-08 & 8.1 \mathrm{E}-09 & 3.5 \mathrm{E}-08 & 1.3 \mathrm{E}-08\end{array}$

For the Struve functions another term is required to achieve the desired accuracy, and the results take the form

(5) $\quad \mathbf{H}_{0}(x)-Y_{0}(x)=\frac{2\left(a_{0}+a_{1}(3 / x)^{2}+a_{2}(3 / x)^{4}+a_{3}(3 / x)^{6}\right)}{\pi x\left(1+b_{1}(3 / x)^{2}+b_{2}(3 / x)^{4}+b_{3}(3 / x)^{6}\right)}+\varepsilon(x)$

and

(6) $\mathbf{H}_{1}(x)-Y_{1}(x)=\frac{2\left(a_{0}+a_{1}(3 / x)^{2}+a_{2}(3 / x)^{4}+a_{3}(3 / x)^{6}\right)}{\pi\left(1+b_{1}(3 / x)^{2}+b_{2}(3 / x)^{4}+b_{3}(3 / x)^{6}\right)}+\varepsilon(x)$,

where the coefficients and errors are listed in Table 6.

TABLE 6

Coefficients of the rational fractions (5)-(6)

$$
\mathbf{H}_{0}(x)-Y_{0}(x) \quad \mathbf{H}_{1}(x)-Y_{1}(x)
$$

$\begin{array}{lll}a_{0} & 0.99999906 & 1.00000004 \\ a_{1} & 4.77228920 & 3.92205313 \\ a_{2} & 3.85542044 & 2.64893033 \\ a_{3} & 0.32303607 & 0.27450895 \\ b_{1} & 4.88331068 & 3.81095112 \\ b_{2} & 4.28957333 & 2.26216956 \\ b_{3} & 0.52120508 & 0.10885141 \\ |\varepsilon|< & 8.2 \mathrm{E}-09 & 2.5 \mathrm{E}-08\end{array}$


Acknowledgement. This work was supported by the Office of Naval Research.

Department of Ocean Engineering

Massachusetts Institute of Technology

Cambridge, Massachusetts 02139

1. M. Abramowitz \& I. A. Stegun, Handbook of Mathematical Functions (AMS 55), Government Printing Office, Washington and Dover, New York, 1964.

2. E. E. AlLEN, “Analytical approximation,” MTAC, v. 8, 1954, pp. 240-241.

3. Y. L. LuKE, Approximation of Special Functions, Academic Press, New York, 1975.

4. C. W. Clenshaw, Chebyshev Series for Mathematical Functions, National Physical Laboratory Mathematical Tables, Vol. 5, Her Majesty's Stationery Office, London, 1962.

5. J. F. HaRT, et al., Computer Approximations, Wiley, New York, 1968.

6. J. G. HAYES, editor, Numerical Approximation to Functions and Data, Inst. Math. Appl., Athlone Press, London, 1970.

7. F. S. Acton, Numerical Methods that Work, Harper \& Row, New York, 1970. 